Revista Prácticum, 2(1), Enero-junio, 2017

\title{
El blog: herramienta de comunicación en las prácticas de enseñanza
}

\section{Blogs: a tool for communication in teaching practice}

\section{Dr. Jesús Casado-Rodrigo.}

Universidad de Sevilla, España

jcasador@us.es

Dra. Rosario Ordóñez-Sierra.

Universidad de Sevilla, España

rordonez@us.es

Casado-Rodrigo, J., Ordóñez-Sierra, R. (2017). El blog: herramienta de comunicación en las prácticas de enseñanza. Revista Prácticum, 2(1), 80-96. 


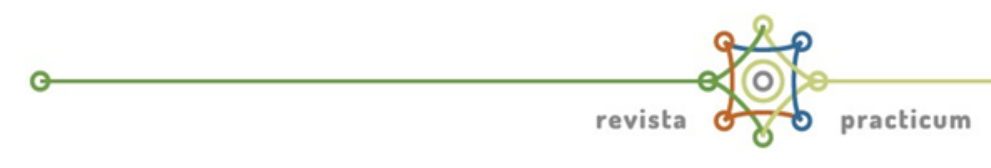

\section{Resumen}

Este estudio plantea cómo viven los futuros maestros de primaria la experiencia de sus prácticas de enseñanza, y cómo construyen su identidad docente y su conocimiento práctico en un contexto intercultural, en especial en cuanto a la enseñanza de inglés como lengua extranjera. Mediante un estudio de caso con un enfoque interpretativofenomenológico, se analiza el blog elaborado por 20 estudiantes de la Universidad de Sevilla durante sus prácticas en colegios de Sevilla y Liverpool. La utilización de un blog poseía el doble objetivo: por un lado, incrementar y mejorar el seguimiento de los estudiantes durante sus prácticas; y por otro, conocer de qué forma vivían los estudiantes la experiencia de sus prácticas de enseñanza, y cómo esta experiencia contribuía a su formación como futuros docentes. Los resultados del análisis revelan un fuerte componente afectivo en el proceso de socialización profesional; así como, una gran desconexión entre las prácticas y la formación inicial en la universidad. La principal conclusión incide en la valoración positiva del uso del blog, ya que muestra la utilidad del blog como espacio narrativo e interactivo; así como (pese a la predominancia de lo afectivo/intuitivo sobre lo técnico/reflexivo) su potencial como herramienta de comunicación y motor de reflexión.

\section{Abstract}

This study discusses how future primary teachers live the experience of their teaching practice internship, and how they build their teaching identity and practical knowledge in an intercultural context, particularly in terms of teaching English as a foreign language. Through a case study with an interpretative-phenomenological approach, this paper analyzes the blog developed by 20 students from the University of Seville during their placement in schools in Seville and Liverpool. A dual objective was addressed with the use of a blog: first of all increasing and improving the process of monitoring and assessing student-teachers during their internship, and also finding out how they lived their experience and how this experience contributed to their training as future teachers. The analysis results bring out the strong role of affective components in the process of professional socialization, as well as the presence of a wide gap between their classroom practice and their initial training studies at university. The main conclusion of this study stresses a positive assessment of the use of blogs, as it shows their usefulness as a narrative and interactive space, and their potential as a communication tool to 
promote reflection (despite the predominance of affective/intuitive elements over technical/reflective ones).

\section{Palabras claves}

Blog, inglés, formación inicial, prácticas de enseñanza, diarios de clase, Programa Liverpool

\section{Key words}

Blog, EFL, initial training, teaching practice, teaching journal, Liverpool Program.

\section{Justificación}

En nuestra experiencia docente en la Facultad de Ciencias de la Educación de la Universidad de Sevilla, la tutorización de prácticas de enseñanza de futuros maestros de primaria supone la oportunidad de estar en contacto con los estudiantes y visitar los centros, llegando a conocer de manera global los diferentes contextos escolares, pero esta visión global queda limitada al tutor y no forma parte de la experiencia de los propios estudiantes, ya que cada uno llega a conocer solo su colegio de manera aislada y las reuniones con el tutor no suelen dar ocasión de compartir más allá de un repertorio de anécdotas que los estudiantes están ansiosos por compartir, tratando de romper el aislamiento que sienten durante las prácticas. En este formato las tareas de tutorización suelen dejar la sensación de que el seguimiento de los estudiantes es insuficiente y poco eficaz $y$, por encima de todo, de que no se consigue que los estudiantes se impliquen en un proceso de reflexión sobre su práctica docente en el que conecten de una forma efectiva las teorías aprendidas con la realidad del aula. La invitación realizada en su momento por el Vicedecanato de Relaciones Internacionales para coordinar y supervisar el Programa de Liverpool se presentó como una valiosísima oportunidad de controlar muchos aspectos del diseño y preparación del programa de prácticas, y de mejorar los procedimientos y la intensidad del seguimiento a los estudiantes.

El Programa de Liverpool, en el que 20 estudiantes de Educación Primaria de la Universidad de Sevilla realizaban sus prácticas de enseñanza de último año en colegios de Sevilla y en colegios de Liverpool, se desarrolló como fruto de un convenio entre las Universidades de Sevilla y de Liverpool Hope, al amparo de un Memorándum de Entendimiento firmado en 2003 por los Departamentos de Educación de los Gobiernos de España e Inglaterra y coordinado por el Instituto Superior de Formación del Profesorado de España y la Teacher Development Agency de Inglaterra. Las prácticas en Sevilla eran de especialidad y seguían el 


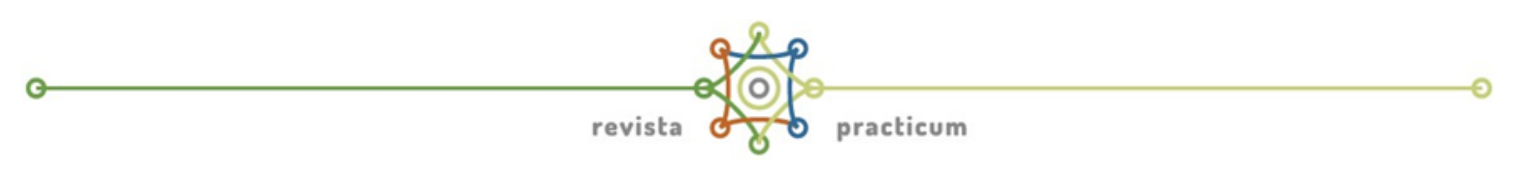

mismo esquema de las prácticas regulares, mientras que en Liverpool eran de carácter generalista y todos los estudiantes se implicaban en planificar e impartir clases de diversas áreas en inglés (primera lengua en las aulas de Liverpool). Los estudiantes impartían sesiones en inglés de áreas tan diversas en la práctica como matemáticas, educación física, lengua o ciencias naturales.

Durante la estancia en los colegios tanto de Sevilla como de Liverpool, debían elaborar diarios de clase en un único blog compartido, en el que todos estaban registrados como autores; se utilizó para ello la herramienta Wordpress. Al igual que en el proyecto de Potter y Banaji (2012) se estableció como requisito que cada estudiante debía escribir al menos dos entradas semanales en el blog y se animó a que también leyeran regularmente las entradas de sus compañeros y añadieran a ellas los comentarios que creyeran pertinentes.

\section{Introducción}

Las prácticas de enseñanza suponen un momento crucial en la formación inicial de los docentes ya que deberán integrar los conocimientos y las teorías pedagógicas adquiridas en la universidad con la realidad de la práctica docente en las aulas; esto supone un proceso de profesionalización, que debe resultar en el desarrollo de pensamiento práctico, al tiempo que constituye un proceso de socialización en la vida y la cultura de la escuela, que contribuirá de forma esencial a la construcción de la identidad docente de los futuros maestros. El prácticum debe desarrollar, a partir de la reflexión sobre la actuación, un pensamiento práctico que ponga en relación la teoría y la práctica, e iniciar al alumno en la socialización profesional (Castaño et al., 1996). Son múltiples los estudios que se centran en el estudio de las prácticas de enseñanza (Domingo, 2008; Gómez-Torres, 2000; González Garcés, 2008; González Garcés y Fernández Prieto, 2009; Gutiérrez, 2001). Para indagar sobre los procesos de socialización y construcción de la propia identidad docente, el enfoque biográfico-narrativo cobra una especial relevancia, no solo como procedimiento de investigación sino como herramienta para promover el pensamiento reflexivo, y también como el formato de la construcción misma de la identidad (Vaillant, 2007). En relación con este enfoque, encontramos estudios centrados en el pensamiento en voz alta, relatos escritos, diarios, cuestionarios 0 entrevistas (Clark y Peterson, 1984), y entre los resultados de diferentes investigaciones (Blázquez y Tagle, 2010; Cabaroglu y Denicolo, 2008; Horwitz, 1985; Johnson, 1992) se señala la práctica reflexiva como instrumento para mejorar la práctica docente.

Para comprender cómo se construye el conocimiento de los docentes y cómo se desarrolla su identidad como tales "es fundamental analizar el modo en que las experiencias personales y las narrativas que se construyen sobre estas actúan en la conformación de esos dos procesos" (Granado y Puig, 2015: 45). El hecho mismo de la escritura es 
un proceso activo y personal que nos hace navegar entre imágenes, poner en palabras una experiencia implica "regresar a esta, hacerla consciente y realizar el esfuerzo cognitivo de elaborar un mensaje adecuado para que pueda ser un acto de comunicación entre quien lo escribe y quien lo lee" (Rekalde, 2009:111).

Por todo ello, para este estudio nos decantamos por el uso de los blogs, ya que desde comienzos de este siglo son una herramienta muy popular y hoy día su uso está plenamente extendido. Casero-Ripollés (2012) corrobora que los jóvenes, a medida que van madurando, registran un aumento en el uso de la mayor parte de los soportes digitales (diarios de pago, radio, blogs, redes sociales, teléfono móvil). La escritura en un blog tiene en su dimensión individual el mismo potencial de un diario reflexivo, convirtiéndose en un artefacto capaz de "potenciar la reflexión de cada estudiante sobre las competencias que adquiere en el contexto de sus prácticas" (Cano, Portillo y Puigdellívol, 2014: 13). Además, el uso de blogs como soporte para los diarios docentes puede aportar características adicionales de interacción y aprendizaje colaborativo (Marcelo, 2011), ya que "permite dar a conocer la propia práctica y contrastarla con la de los colegas, estableciendo redes de apoyo mutuo y de revisión colegiada de la práctica" (Portillo, Cano y Giné, 2012: 67).

En nuestro contexto, los estudiantes durante las prácticas se implican en una inmersión completa en el contexto del centro al que son asignados, separándose de sus compañeros y viviendo su experiencia de forma aislada, una experiencia que tiene un fuerte componente afectivo y de socialización profesional. Compartir sus experiencias en un blog puede contrarrestar esta sensación de aislamiento; en estudios sobre uso educativo de blogs durante prácticas profesionales se observa que los estudiantes "valoraban positivamente saber que no estaban solos en sus esfuerzos, dudas e inseguridades. Los sujetos escribían sobre retos y dudas que muchos de ellos estaban experimentando, y esta franqueza les ayudaba a reducir el miedo y la ansiedad" (Ladyshewsky y Cardner, 2008: 249).

Durante las prácticas, el blog permite a todo un grupo compartir regularmente sus diarios e interactuar de forma asíncrona; los blogs académicos "añaden una dimensión reflexiva o cognitiva a las narrativas personales, e introducen una dimensión emocional y social al trabajo académico formal que a menudo refuerza las tareas cognitivas o intelectuales" (Deng y Yuen, 2011), lo que abre un amplio abanico de posibilidades para la supervisión, la interacción, el apoyo afectivo y la reflexión compartida.

\section{Metodología}

En el terreno de la investigación se planteó descubrir de qué forma "piensan" (y de qué forma "piensan juntos") en general sobre la experiencia de prácticas y específicamente sobre la enseñanza de lengua 


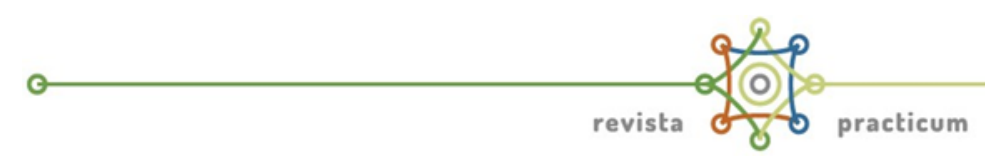

extranjera (L2) un grupo de futuros maestros durante sus prácticas. Por lo tanto, nuestro objetivo consistió en:

Conocer cómo viven los estudiantes las prácticas de enseñanza y en qué medida las prácticas suponen una experiencia significativa en el proceso de construcción de su identidad docente en relación con la enseñanza de Lengua extranjera.

Se optó por un estudio instrumental de caso único (Stake, 1995): el Programa Internacional de Prácticas de Magisterio en Liverpool; cada estudiante se constituiría en una unidad de análisis integrada en el caso (Yin, 2004). Los criterios que llevaron a la selección de este caso, fueron (1) la motivación y la inquietud de los estudiantes por participar en un Programa de prácticas innovador, (2) las características del Programa, que permitiría observar la influencia en futuros maestros de inglés de un contexto escolar angloparlante, y (3) la interacción que originaba el desarrollo mismo del Programa, que daba como resultado una cierta cohesión y sentido de pertenencia al grupo entre los estudiantes.

La población de la que procede el grupo de participantes en el estudio la componen los estudiantes de último curso de la extinta Diplomatura de Magisterio que cursaban la asignatura Prácticas II. Los estudiantes procedían de cualquiera de las especialidades existentes; de los 20 seleccionados 13 eran especialistas en Lengua Extranjera, 2 en Educación Física, 2 en Educación Primaria, 2 en Educación Infantil y 1 en Educación Especial. El grupo estaba compuesto por 15 mujeres y 5 hombres.

El medio de producción y recogida de datos para la investigación fue principalmente el diario de prácticas en formato blog. Se pensó en un diario post instructivo donde los estudiantes pudieran describir cómo se desarrollaban sus experiencias y pudieran debatir sobre sus impresiones. Este instrumento se integra en la experiencia con varias funciones: la principal del diario, que es dotar a los estudiantes de un espacio de expresión y reflexión; también debía permitir la interacción entre los estudiantes de modo que pudieran comentar sus experiencias y brindarse apoyo, constituirse en una herramienta para el seguimiento del profesorado universitario, y registrar todas las entradas y comentarios de forma estructurada para poder acudir a ellos cuando fuera necesario, tanto para fines de docencia como de la investigación.

Los datos obtenidos son fundamentalmente descriptivos, tratándose de las narraciones que los estudiantes hacen en los diarios de su experiencia de prácticas. El análisis del contenido de los diarios permitió elaborar una descripción de la experiencia de prácticas de enseñanza desde el punto de vista de los estudiantes. Para mantener el punto de vista, desde una perspectiva cualitativa, se trató de identificar las interpretaciones clave de los sujetos estudiados (Erickson, 1986). 


\section{Resultados}

En teoría, las prácticas deberían suponer el momento en que los estudiantes actualicen y pongan a prueba, en un contexto real y bajo la supervisión y orientación de sus Tutores Profesional y Académico, toda la formación que han recibido durante la carrera. Sin embargo los diarios dibujan una situación muy diferente: de algún modo, los estudiantes llegan a los colegios pensando que se incorporarán a un aula y serán capaces de desplegar su sabiduría, la adquirida en la Facultad, sin ningún obstáculo. El encuentro con la realidad del aula, la interacción con los alumnos y la puesta en juego simultánea del complejo conjunto de elementos que conforman el currículo los sorprende y los disgusta, ya que se pone de relieve su impericia y su falta de competencia profesional, de lo que culpan a la Facultad, que no les ha enseñado bien, e incluso al Tutor Profesional, que no les brinda suficientes posibilidades de aprendizaje en la práctica.

Dado que su acervo de experiencias puramente profesionales es muy limitado, los estudiantes establecen un nexo con sus vivencias escolares para dar significado a las situaciones a las que se enfrentan, dando una visión muy general, bastante romántica, de su vocación, con un fuerte componente afectivo y de nostalgia de infancia.

"No sabría decir porque, pero mi colegio huele a mi infancia. Supongo que parte de culpa tiene haber estado 13 años en un colegio de monjas y después dos años en uno de curas, pero es que parece que en Santa Isabel hay una mezclilla de los colegios de mi infancia, y la verdad me siento como en casa". CAR 27-ene

Uno de los efectos de esto es que recuperan ejemplos de acción docente desfasados, pasando por alto los modelos estudiados.

"Yo creo que no es malo que tengan pies, hay costumbres "tradicionales" que no están mal que se mantegan, tampoco todo es malo. Me parece estupendo mantener el orden y el respeto a los demás, y lo de tener piernas, más que nada para las espaldas de esos niños... digo yo". ANA en PAU 28-ene 3/12

En contraste, los estudiantes tienen por lo general una opinión bastante deficiente de la formación inicial: estiman que esta formación teórica no se adecua a las necesidades que les plantea la práctica, atribuyen externamente su frustración y categorizan la teoría aprendida como irrealizable y utópica.

"Nos examinamos de mucha teoría (que mucha de ella parece que no te sirve casi para nada), la "vomitamos" en el examen y "si te he visto no me acuerdo". Y lo que de verdad importa, como cómo 


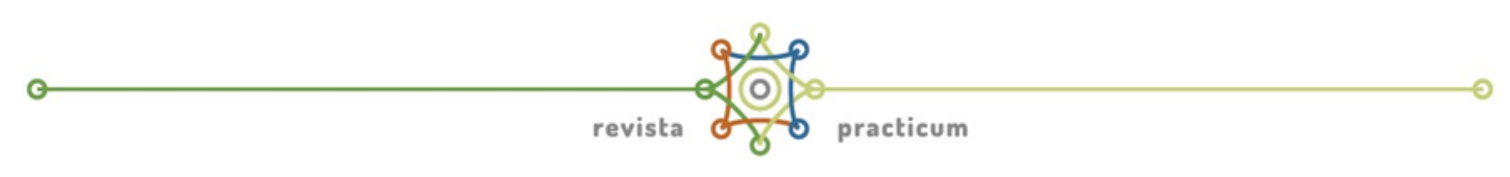

trabajar con un niño a quien le sucede " $x$ ", o cómo relacionarnos con los padres para que estos se impliquen en el aprendizaje de sus niños... de todo eso nos enseñan lo mínimo, mínimo. Y lo poquito que vemos, a menudo es superficial. Nos pintan, como bien decís, los casos y las clases "ideales" y ya está". SON en JUA 28-ene $12 / 15$

Inmersos en el contexto de las prácticas, los estudiantes están más preocupados por su propio desempeño ante los retos que les van a plantear sus intervenciones en el aula (la "práctica") que por el análisis crítico de las mismas (la "teoría"). Por lo general suelen ser bastante benévolos consigo mismos y se sienten satisfechos con sus actuaciones.

Las narraciones de los estudiantes reflejan también la relación que mantienen con los Tutores Profesionales, a quienes rara vez parecen percibir como su instructor o supervisor; más bien los perciben como una más de las circunstancias de un contexto fijo al que de alguna forma se sienten sometidos, con poca posibilidad de cambio o intervención por su parte.

\begin{abstract}
"no sé muy bien cómo enfocar mis clases porque se supone que el profesor me deja "libertad" (al menos eso dice él), pero luego me dice que me ajuste al tema y al libro, que no me desvaríe demasiado porque los niños ya están acostumbrados a una forma de trabajar (a SU forma, vaya)". SON 1-feb
\end{abstract}

De esta forma, perciben la actuación de los maestros con un cierto determinismo como un modelo de lo que podría ser su propio desempeño profesional en el futuro, al tiempo que expresan con cierta pasión una idea de "huida hacia adelante": el compromiso de que en el futuro, cuando estén a cargo de sus propios grupos y sean "maestros completos", podrán poner en práctica modelos mejores.

"Cuando llegó la profesora sin tener ni idea de qué hacer e improvisó. Empezó la clase cantando la canción (que los niños ya se la saben de memoria y es la única que se saben en inglés) y después encontró allí carteles de colores de la clase de español y decidió decirlos en inglés. Me refiero con esto a que la improvisación cuando no se sabe qué hacer es a lo que todos recurren y seguramente recurriremos!". LEO en INE 27-ene 8/10

En general se sienten bien recibidos por los alumnos, pese a los muchos miedos, en gran medida inespecíficos, que traen consigo. Pero la inseguridad que les provoca el que los alumnos puedan no verlos como "maestros de verdad" les lleva a adoptar actitudes un tanto represivas y a dar demasiada importancia a la disciplina como forma de ganar el respeto que creen que el alumnado les niega al cuestionar su rol docente, en 
lugar de tratar de ganarse este mismo respeto mejorando sus prácticas docentes.

"La idea central que quiero transmitir en este texto es la importancia de la disciplina en clase, y del respeto a la figura del profesor. Si queremos ser buenos docentes, tenemos que guardar las distancias con nuestros estudiantes, dejando siempre claro que nosotros somos los profesores y ellos los alumnos". DAM 14-febB

La mayor parte de sus inseguridades y miedos se refieren a la falta de disciplina por parte de los alumnos y a la necesidad de poner orden para poder avanzar con la actividad en curso y están fuertemente conectados con la percepción que piensan que el alumnado tiene de ellos. Sienten que están siendo constantemente puestos a prueba por los alumnos, que activamente se dirigen a ellos con la intención de comprobar sus límites, exponer sus debilidades y, en definitiva, ponerlos en evidencia. En este sentido hacen una narración del alumnado como si del enemigo se tratase.

"Primero de tantean haciéndote un montón de preguntas con el fin de ver como reaccionas. A mí por ejemplo, el jueves pasado dí mi primera clase con un $5^{\circ}$. Se me acercó una niña y me habló en francés del tirón ( $\mathrm{mi}$ cole es que es bilingüe de francés). Entendí lo mínimo pero lo que hice fue mandarla a su sitio y decirle que estábamos en clase de educación física. (en realidad me dejó tela de cortá) pero bueno salí airosa porque mostré seguridad con mi respuesta". DOR 2-feb

Al incorporarse a los colegios de Liverpool aparecen en el blog numerosos comentarios en cuanto a sus problemas de comunicación en inglés, que ahora es la primera lengua de uso universal (aunque extranjera para los estudiantes). En los diarios correspondientes a la estancia en colegios de Sevilla no se cuestionaron sus posibles carencias en cuanto a conocimientos, nivel o competencia comunicativa en inglés, y al mismo tiempo se mostraban muy críticos con el nivel de inglés de sus Tutores Profesionales; ahora sin embargo su pobre competencia comunicativa en inglés, con los conflictos que ello les ocasiona dentro y fuera de las aulas, se convierte lógicamente en uno de los temas estrella, influyendo como factor decisivo en su desempeño en las aulas y en su relación con sus Tutores Profesionales.

"Creía que iba a ser más fácil, pero el hecho de meterte de lleno en un sistema educativo que no conoces no es tan fácil como yo creía. Tuve muy claro desde que entré en la carrera que quería haces las prácticas aquí. De hecho no se me fue de la cabeza en estos 3 años... pero está claro que no lo había pensado con suficiente frialdad. Ahora que estoy aquí, veo claro que ni de lejos es fácil 


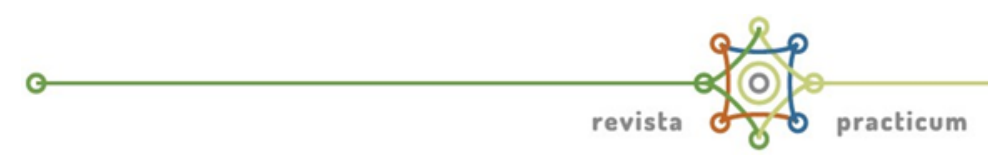

integrarse en un país extranjero. Diferentes horarios, otro idioma (el cual te das cuenta que no dominas tan bien como creías), otra forma de vida, no familia, no amigos... Y mucho menos en un colegio...(de hecho tampoco es fácil integrarse en un colegio español)". CAR 11-mar

Las primeras experiencias en los colegios vienen marcadas, para casi todos, por el idioma, y son frecuentes las referencias al "scouse", el fortísimo acento dialectal de la zona de Liverpool. En estos problemas con el acento local (y en general con el idioma), los estudiantes perciben la importancia de que en la interacción se dé por ambas partes una intención clara de resolver los problemas de comprensión y un esfuerzo mutuo por comunicarse. Es decir, viven la experiencia de que la comunicación es, en efecto, un proceso colaborativo.

"...mi tutora también tiene un acento muy cerrado y muy fuerte, pero ella misma me ha dicho que me va a hablar más lento, porque sabe que no es la que mejor habla...jejejeje". LEO en ROS 24-feb $3 / 3$

Lógicamente, los problemas con el idioma condicionan fuertemente la percepción que tienen de los colegios a los que se incorporan y su proceso de integración en el centro y en el aula. En este sentido, juega un papel muy relevante la sala de profesores ("staff room"), que los estudiantes perciben como lugar emblemático donde comprueban su nivel de integración en el colegio.

"como te entiendo...en mi staff room si hablan, pero entre ellas, a mi me dejan a un lado o tan solo se dirigen a mi para decirme "a tee or coffe?" y a lo mjr cuando yo hablo para integrarme m sueltan un "ahhh" y su snrisa... se echa de menos decir, ique calor hoy! jajaja (esque ahí ni si quiera se habla del tiempo, que es tan tipico cuando no hay nada que hablar)". LEO en CAR 1-mar 1/10

Los problemas de comunicación relacionados con el idioma adquieren un carácter especial cuando se trata de los alumnos en el aula. No ser capaces de comprender a los niños les preocupa especialmente, y se animan unos a otros en este sentido.

"El martes pasado o segundo día de mis prácticas en Inglaterra, mientras jugaban todos los niños, tanto los de 'Nursery' como los de 'Reception', una niña me preguntó de repente: - "Miss, is it tidy up time?" Yo no la entendí, para que nos vamos a engañar. No tenía más de cuatro años $y$, entre esa vocecita de niña que me trae, acompañado de este acentazo "scouse" /'skaus/, y seguido de mi segundo día rodeada de profesores, niños y secretaria ingleses de los cuales ni uno tiene idea de español, yo no sabía qué narices me 
estaba preguntando la pobre chiquilla. Así que le dije una de mis frases más usadas en el colegio 'Ask the teacher' ". SON 28-feb

Por otro lado se dan cuenta de que su nivel de conocimientos está efectivamente por debajo del de los alumnos, en especial en las sesiones de lengua ("literacy"), donde los alumnos desarrollan competencias específicas en el uso de inglés como primera lengua, con lo que de alguna forma los estudiantes acaban sintiéndose "alumnos" ellos mismos, ya que tienen que aprender lo mismo y al mismo tiempo que los niños.

Sin embargo, lo que podría haber resultado una experiencia catastrófica también se va convirtiendo en tarea difícil pero superable, en gran medida gracias a la ayuda que reciben del Tutor Profesional y también de los mismos alumnos: una nueva ocasión para entender, esta vez dentro del aula, que la comunicación (es decir, toda interacción que tiene lugar en el aula) es básicamente un proceso colaborativo.

"La verdad que la maestra se ha portado muy bien conmigo porque les pidió que me ayudaran a escribir la palabra, me la deletreaban, les explicó que como yo era d otro país y demás..." ELE 9-mar

A la hora de impartir sesiones en inglés, los problemas de lengua se multiplican. En muchos casos dan a entender, aunque no siempre de manera explícita, la ansiedad con que se enfrentan a dar clase de inglés en inglés a niños ingleses, una presión que en efecto les empuja a ponerse en el rol de alumno y alejarse del rol de docente.

Pronto los estudiantes comienzan a ejercer de embajadores en sus sesiones de español y despliegan, en contraste con la cultura "scouse", todo su repertorio de flamenco, sevillanas, mantoncillos, castañuelas, abanicos y tortilla de patatas para regocijo de los alumnos ingleses (y de sus maestros). Por lo general los estudiantes explican que los alumnos se han sorprendido y se han divertido mucho con todo esto, pero también los estudiantes se ríen con la pronunciación y el desempeño de los niños ingleses en las actividades relacionadas con el español y la cultura.

"Yo ayer los enseñé a decir iiiqué frío hace!!! jajajaja y tendríais que ver a toda una fila de niños de 5 años ingleses diciendo q frío con acento "scouse" y haciendo como q tiritaban a la vez jajajaja". PAU en GAL 24-feb 3/4

Las narraciones que hacen de sus intervenciones en el aula suelen aludir por lo general a los nervios y la inseguridad con que las afrontan, aunque después de dar su clase tienen una visión generalmente positiva, en la medida en que han conseguido superar la prueba. Son frecuentes las menciones a los nervios, al miedo a enfrentarse a la clase $y$, a veces, también a hablar inglés. 


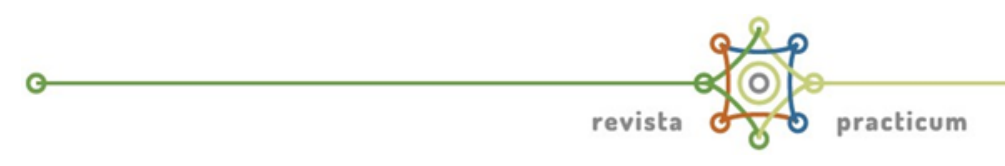

"Hoy estaba aterrorizada porque tenía que dar dos clases, a última y a penúltima hora, a dos $6^{\circ}$. Temía que la clase fuera un caos, porque ya a esas horas los niños casi no atienden". LAR 4-feb

En la mayoría de los casos estas narraciones son meramente descriptivas, carentes de reflexión o incluso de una narración de resultados. Esta ausencia de profundidad crítica suele ir acompañada de un tono muy positivo: todo fue bien, a todos les gustó, estoy muy satisfecho. Los aportes de los compañeros suelen ser simples refuerzos positivos, alegrándose por las experiencias adquiridas. Es a partir de esta sensación de "prueba superada" como van construyendo su imagen de docente.

Las narraciones en el blog sobre las sesiones observadas por el Tutor Académico no tienen el mismo tono positivo. Ahora aparecen expresiones como "debería haber hecho tal o cual cosa", a modo de justificación por aquello que saben que no han hecho del todo bien. Los estudiantes saben de sus limitaciones aunque eviten hablar de ellas. Puede parecer en principio que la reflexión crítica ha de ser inducida por un observador o un agente externo, pero aunque en cierta medida sea así, parece probable que el proceso de reflexión sea más bien íntimo.

\section{Discusión y conclusiones}

Nos proponíamos descubrir a través de sus aportaciones en el blog cómo la experiencia del prácticum, la interacción entre los estudiantes y el contexto intercultural contribuían a la construcción de su identidad docente en relación con la enseñanza de lengua extranjera. Básicamente encontramos una gran desconexión entre su formación universitaria y su experiencia en los colegios (lo que les lleva a conectar más con ideas sobre la enseñanza de L2 procedentes de su biografía escolar previa) junto con la fuerte carga afectiva con que viven todo el proceso de las prácticas.

En cuanto a la interacción entre los estudiantes, el blog como herramienta colaborativa ha funcionado, como era su objetivo, para poner a cada estudiante en contacto con experiencias y puntos de vista más allá de su experiencia individual y permitir la identificación de contrastes y situaciones de conflicto. Sin embargo, en la línea de otros estudios sobre niveles de reflexión (Dos y Demir, 2013; Roca y Manchón, 2007), la reflexión conjunta en la interacción que aparece en los diarios es escasa, tanto en cantidad como en profundidad. Algunas entradas individuales son más reflexivas que el debate que se produce a continuación en los comentarios. Puntualmente se aprecia un mayor nivel reflexivo y técnico también en los comentarios.

Los estudiantes viven el periodo de prácticas con mucha intensidad, como una etapa importante hacia su profesionalización, a caballo entre sus estudios iniciales y su futuro como docentes, y como campo de 
experimentación donde poner a prueba sus competencias y los recursos adquiridos durante su formación. En efecto, este momento debería servir como proceso de socialización en el contexto escolar por un lado y como proceso de integración de los recursos adquiridos en la universidad con la práctica efectiva en el aula por otro, contribuyendo ambos a la formación de su identidad docente y de su pensamiento práctico, pero estos dos procesos no parecen funcionar con la misma intensidad ni al mismo nivel. Podemos ver cómo viven todos los momentos de la experiencia como un reto personal con una fuerte carga afectiva, y es en estos términos como van comprobando cómo encajan en el rol y van ensayando sus competencias.

Básicamente se enfrentan al reto de "ser maestros": sentirse maestros y actuar como tales, desempeñando el rol para el que supuestamente se han estado preparando en la universidad; pero este reto lo afrontan con bastante inseguridad y desde una posición pobremente definida, ya que se sienten como alumnos (completando sus estudios para ser evaluados) pero querrían ser percibidos en el aula como maestros, especialmente por los alumnos, lo que parece ser un requisito para llegar a sentirse maestros ellos mismos. La imagen de maestro que construyen en los colegios está muy vinculada con su experiencia vital y con su propia biografía escolar, frente a la que el conocimiento académico que adquirieron en la universidad adquiere un "carácter efímero" (Pérez Gómez, 1999).

Durante las prácticas la Facultad les resulta algo lejano y desconectado de las situaciones a las que ahora se enfrentan. Valoran pobremente la formación recibida durante la carrera por suponerla esencialmente "teórica" y porque sienten que no les ha aportado referentes para interpretar la realidad que se encuentran en el aula ni competencias para intervenir en ella, lo que supone un obstáculo más ante el reto que supone "hacerse maestro" en las prácticas.

En los diarios se identifican numerosas situaciones de conflicto susceptibles de provocar reflexión, y que de hecho provocan debate.

En los diarios, lo afectivo/intuitivo se impone claramente a lo técnico/reflexivo. Esto resulta evidente en cuanto a la interacción en el aula con los alumnos, que son percibidos a la vez como adversarios y como la fuente de gratificación que les consolida en su rol. Frente a esto, la formación inicial parece superficial y difusa y los estudiantes tratan de superar el reto del prácticum con los pocos recursos con que parecen contar, con frecuencia orientados por la imagen de maestro vocacional y por la concepción de la lengua extranjera como una materia académica más, ideas que ya habían construido durante todos sus años escolares previos a la universidad y que su experiencia en las prácticas les refuerza.

En efecto los estudiantes ven la L2 como una materia académica más, y la función del maestro es "enseñarla" en el aula, aunque no parecen tener claro qué es eso de "enseñar" una lengua. Resulta representativo por ejemplo el análisis de sus posiciones en cuanto al uso de la primera lengua en el aula de lengua extranjera. En principio traen 


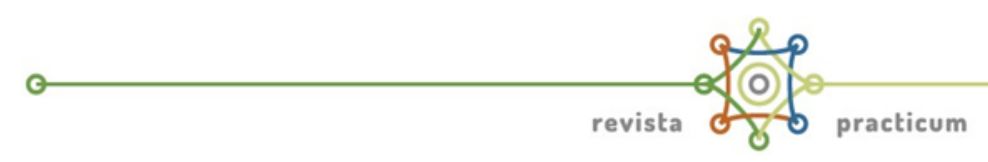

de la universidad un modelo que establece que las clases de inglés deben ser enteramente en inglés, y tratan de hacerlo así, pero en los colegios de Sevilla este modelo inicial entra en conflicto con la práctica de los tutores profesionales y con el hábito adquirido por los alumnos (que usan sistemáticamente la primera lengua en el aula de L2), lo que les causa bastante ansiedad y frustración, y tienden a descartar el modelo por inviable, aunque deseable. Mantienen un buen número de debates en los diarios a este respecto, aunque no hacen mención a su propia competencia comunicativa ni a cómo sus posibles carencias puedan influir en su ansiedad; reflejan una cierta adhesión a modelos académicos obsoletos de enseñanza formal de la $L 2$, al tiempo que expresan una confianza difusa en que llegarán a dar clases enteramente en inglés en el futuro, cuando estén a cargo de sus propias aulas.

Durante su estancia en Liverpool, los estudiantes parecen desenvolverse en el contexto inglés a un nivel meramente monocultural (Meyer, 1990), con una percepción superficial de los contrastes interculturales. Por otra parte, el uso del idioma les ocasiona problemas de comunicación que interfieren en dos aspectos fundamentales: (a) sus procesos de socialización e integración en la comunidad escolar, y (b) la interacción en el aula con tutores y con alumnos. En los dos casos son conscientes de la ayuda que reciben tanto del resto de maestros como de los propios alumnos, y de cómo, a pesar de sus carencias con el idioma, la comunicación es posible en la interacción gracias a la colaboración activa de ambos participantes

La incorporación del blog al programa de prácticas ha servido para que los estudiantes expresen libremente sus vivencias, y esto lo valoramos muy positivamente. Sin embargo, al llegar a las prácticas, los estudiantes ya deberían contar con una formación en la cultura de lo comunicativo para experimentarla en sus aulas y también para enriquecer su interacción con sus iguales; como señalan López-Vidales \& GonzálezAldea (2014) y Dillenbourg (2012) la corresponsabilidad y el aprendizaje colaborativo desarrollados en su formación no garantizan por sí mismos su eficacia por el mero hecho de utilizarlo

\section{Referencias}

Blázquez, F. \& Tagle, T. (2010). Formación docente: un estudio de las creencias de alumnos y profesores sobre el proceso de enseñanza y aprendizaje del inglés. Revista Iberoamericana de Educación, 54(4), 1-12.

Cabaroglu, N. \& Denicolo, P. (2008).Exploring student teacher belief development: An alternative constructivist technique, snake interviews, exemplified and evaluated. Personal Construct Theory \& Practice, 5, 2840. Recuperado dehttp://www.pcpnet.org/journal/pctp08/cabaroglu08.html. 
Cano, E., Portillo, M.C. \& Puigdellívol, I. (2014). Evaluación de competencias en los entornos de prácticum de los estudios de magisterio mediante el uso de blogs. Tendencias Pedagógicas, 26, 9-28.

Casero-Ripollés, A. (2012). Más allá de los diarios: el consumo de noticias de los jóvenes en la era digital. Comunicar, 39, 151-158. doi: 10.3916/C39-2012-03-05.

Castaño, N., Prieto, C., Ruiz, E. \& Sánchez, M. (1996). El profesor tutor del Prácticum: Propuesta de modelo. Revista Electrónica Interuniversitaria de Formación del Profesorado, 1(0), 1-5. Recuperado de http://dialnet.unirioja.es/servlet/articulo?codigo $=2786700$

Clark, C.M. \& Peterson, P.L. (1984). Teachers' thought processes. Occasional Paper, 72. East Lansing, MI: Institute for Research on Teaching, Michigan State University. Recuperado de http://eric.ed.gov/?id=ED251449

Deng, L. \& Yuen, A.H.K. (2011). Towards a framework for educational affordances of blogs. Computers and Education, 56, 441-451.

Dillernbourg, P. (2012). Classroom Ergonomics. Jornada de Aprendizaje Colaborativo en Entornos Virtuales. Barcelona 26-27, noviembre. Recuperado de (www.youtube.com/watch?v=tdF_AnyiYfk) (28-03-2013).

Domingo, A. (2008). La práctica reflexiva en la formación inicial del maestros/as. La evaluación de un modelo. (Tesis de doctorado). Universitat Internacional de Catalunya.

Dos, B., \& Demir, S. (2013). The analysis of the blogs created in a blended course through the reflective thinking perspective. Educational Sciences: Theory and Practice, 13(2), 1335-1344. Recuperado de http://www.edam.com.tr/kuyeb/tr/makale.asp?ID=850\&act=detay

Erickson, F. (1986).Qualitative Methods in Research on Teaching. En Wittrock, M.C. (ed.), The Handbook of Research on Teaching. New York: MacMillan.

Granado, C. \& Puig, M. (2015). La identidad lectora de los maestros en formación como componente de su identidad docente. Un estudio de sus autobiografías como lectores. Ocnos, Revista de Estudios sobre Lectura, 13, 43-63.

Gómez-Torres, Ma.J. (2001). Un estudio longitudinal sobre el Prácticum en la formación de los maestros de la especialidad de Educación Especial. (Tesis inédita de doctorado). Universidad de Sevilla. 


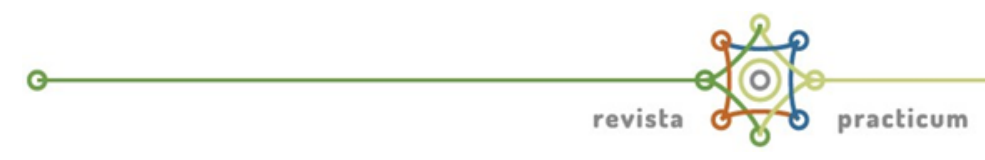

González Garcés, Á.M. (2008). Análisis crítico del prácticum de Magisterio en una facultad de Formación de Profesorado y Educación. (Tesis de doctorado). Universidad Autónoma de Madrid.

González, Á.M \& Fernández, M.S. (2009). Análisis crítico del Prácticum de Magisterio en una Facultad de Formación de Profesorado y Educación. Tendencias Pedagógicas, 14, 417-432.

Gutiérrez, I. (2001). La enseñanza reflexiva en el prácticum de magisterio. Anuario Pedagógico, 5: Educación en derechos humanos, ciudadanía y formación crítica de maestros y maestras. Santo Domingo (República Dominicana): Centro Poveda. Recuperado de http://www.centropoveda.org/IMG/pdf/ensenanzareflexiva.pdf.

Horwitz, E.K. (1985). Using student beliefs about language learning and teaching in the foreign language methods course.Foreign Language Annals, 18(4), 333-340.

Johnson, K.E. (1992). Learning to teach: Instructional actions and decisions of preservice ESL teachers. TESOL Quarterly, 26(3), 507.

Ladyshewsky, R.K. \& Cardner, P. (2008).Peer assisted learning and blogging: A strategy to promote reflective practice during clinical fieldwork. Australasian Journal of EducationalTechnology, 24(3), 241-257.

López-Vidales,N. \& González-Aldea, P. (2014). Audioblogs y Tvblogs, herramientas para el aprendizaje colaborativo en Periodismo. Comunicar, 42, 45-53. doi: http://dx.doi.org/10.3916/C42-2014-04.

Marcelo, C. (2011). Modelos innovadores en la supervisión del practicum. En Raposo, M., Martínez, M.E., Meyer, M. (1990). Developing Transcultural Competente: Case Studies of Advanced Foreing Language Learners.EnButtjes, D. \& Byram, M. (eds), Mediating Languages and Cultures. Clevedon: Multilingual Matters Ltd., 136-158.

Pérez Gómez, Á.I. (1999). El practicum de enseñanza y la socialización profesional de los futuros docentes. En Á. Pérez Gómez, J. Barquín Ruiz, \& J. F. Angulo Rasco eds., Desarrollo profesional del docente: política, investigación y práctica (pp. 636-660). Madrid: Akal.

Potter, J.\& Banaji,S. (2012). Medios sociales y autogestión del perfil digital: identidad y pedagogía con blogs en un máster. Comunicar, 38, 83-91. http://dx. doi.org/10.3916/C38-2012-02-09.

Portillo, M.C., Cano, E. \& Giné, N. (2012). La escritura de blogs para la evaluación de competencias del prácticum de formación del profesorado. Bordón, 64(4), 63-81. 
Rekalde, I. (2009). ¿Cómo podemos aprender a mejorar nuestras prácticas docentes?. Los diarios del alumnado universitario: herramienta de formación permanente del profesorado. Bordón, 61(4), 109-122.

Roca, J. \& Manchón, R.M. (2007). Análisis de la reflexión y preparación para la actividad reflexiva en el prácticum de maestro (Inglés). Revista de Educación, 342, 373-396.

Stake, R. E. (1995). The Art of Case Study Research. Londres: Sage.

Vaillant, D. (2007). La identidad docente. I Congreso Internacional Nuevas Tendencias en la Formación del Profesorado. Barcelona: Grupo de Trabajo sobre Desarrollo Profesional Docente en América Latina. Recuperado de https://goo.gl/ntETIL

Yin, R.K. (2004). The Case Study Anthology. Londres: Sage. 